\title{
Analysis and forecasting of performance indicators of air transport facilities
}

\author{
Elena Kochkina, Elena Radkovskaya and Kseniya Denezhkina \\ Ural State University of Economics, 8 Marta Str., 62, 620144 Ekaterinburg, Russia
}

\begin{abstract}
Aviation is one of the most significant growth drivers not only for the transportation infrastructure, but also for country's economy as it contributes to both industrial and social progress of society. The paper focuses on Sheremetyevo International Airport, one of the largest Russian airports and analyzes it in terms of short-term and long-term perspectives. Using real statistical data, the article presents the models for forecasting essential performance indicators of airports, namely, passenger traffic and revenue. By comparing quality characteristics, most suitable models were selected and checked for adequacy. Forecasts of the probable changes in the values of the performance indicators were made under the assumption of maintaining the previously formed development trend.
\end{abstract}

\section{Introduction}

The world crisis caused by the Covid-19 pandemic has highlighted the importance of ensuring sustainable development in territories, both on the local (the level of municipalities), and the global scale (the level of regions and country). A sustainable territory a priori has better chances to quickly and efficiently cope with an emergency. This is reinforced by at least two factors: the established networks between different levels and entities of territorial governance and accumulated experience of managing available resources and reserves, which enables to allocate them in the shortest possible time.

The authors interpret sustainable development in two aspects. At the intra-territorial level, sustainability marks smooth and balanced development of a territory in key social and economic areas, as well as the existing interaction between territorial entities. In this sense, a territory is considered as a relatively autonomous unit, valued on an individual basis. At the external territorial level, each territory is considered as an integral part of a larger area, and in this sense, its development is a constituent of the sustainable development indicator of the whole area. From this point of view, sustainability implies a set of close-to-average-value indicators of social and economic development of all territories, as well as a fairly smoothed trend of progressive development of the area as a whole. In both cases, although at different levels, sustainability is determined not only by entities within the boundaries of the studied area, but also - to a larger extent - by a degree of development of the existing territorial infrastructure.

One of the most obvious infrastructure attributes of a territory is its transport network [1]. At the same time, it is important to assess not only the availability of the essential elements of the network - roads for various types of transport, the fleet of cars, objects that 
ensure the functioning of the logistics chain, etc., but also management mechanisms of the transport system as a whole and its individual components.

Aviation is one of the most significant growth drivers not only for the transportation infrastructure, but also for country's economy as it contributes to both industrial and social progress of society. In Russia, air transport takes its place in the country's transport system, providing up to $20 \%$ of the total passenger traffic, and this importance tends to grow. For long-distance (over $1000 \mathrm{~km}$ ) carriage of passengers, mail and freight shipments, air transport is the predominant means of transportation since it allows to save costs and time per kilometer. It is worth noting that for some remote northern regions of Russia, air transportation is the only alternative [2].

The development of complex network of tourism, as well as the general trend towards globalization, has increased the need for international communication, both in business and culture [3]. Air transport plays a significant role in satisfying this need. Thus, the study of the current state of the aviation industry, established trends, and areas for further development is therefore becoming an urgent and relevant task [4]. Using inductive and deductive approaches to empirical data analysis may lead to correct estimation of air transport facilities of all levels - from a specific airport or airline company to the whole industry.

Considering the air transport industry as an economic system, we focus on one of the Russian airports and analyze it in terms of short-term and long-term perspectives and make an attempt to predict possible changes in the current growth trend by taking into account the airport characteristics.

\section{Materials and Methods}

Forecast of key performance indicators plays an important role in the evolution of theories and applications of regulating economic systems in order to achieve sustainable development. Regulation should ensure the selection and implementation of the most rational decisions, since potential damage from making non-optimal decisions may be quite extensive [5]. By focusing on predicted outcomes, it is often possible to avoid situations that run counter to sustainable development.

In this study, the authors were building the models that could be used for forecasting key indicators of the airport performance, namely, passenger traffic and revenue. The statistical data of Sheremetyevo International Airport were studied. Sheremetyevo International Airport is one of the TOP-10 hub airports in Europe, one of the TOP-50 in the world and the largest Russian airport in terms of passenger and freight traffic. In 2020, Sheremetyevo ranked fifth in Europe with regard to passenger traffic.

The initial estimation of the parameters of the proposed model is based on the study of the initial time series, which is also used for the subsequent adjustment of the obtained parameters. For adaptive forecasting methods, based on new data obtained at each step in time, the model parameters are further adjusted to new, continuously changing conditions. Thus, the model constantly absorbs new information and adapts to it. Since the study time series till 2020 followed a clear trend, i.e. it contained a long-term constituent that showed increasing values of the series over the analyzed period, trend models were also used for predictions.

Economic and mathematical forecasting models for the considered indicators can serve as a useful research tool [6]. However, it is worth noting that in order to improve the accuracy of forecasts for any indicator (including those selected for our analysis), under changing conditions, in periods of uncertainty or incomplete information, some additional work on models is needed. That is why adaptive forecasting methods that reflect the current properties of the dynamic series and are able to take into account the evolution of dynamic 
characteristics of the studied processes. play an important role in the model improvement. Prediction outcomes are the most important condition for regulating the development of economic systems in order to ensure their sustainability [7]. Forecasting helps to identify distinctive patterns of the studied indicator and to develop hypotheses about the most likely directions of its change.

Testing the relevance of the forecasting model is an important stage of research [8]. To obtain the most reliable results, it is necessary to perform a preliminary analysis of the time series and to determine its nature. For this purpose, the deviations between the calculated and actual values have been analyzed. If the shape of the prognostic curve is set correctly, then the named deviations have a normal distribution, sharply deviating values are random, there is no autocorrelation, and the variance of the deviations stays unchanged over time.

A set of values of passenger traffic from 2009 to 2019 was used for forecasting. Initially, the quality survey of the series was performed [9]. The test results related to the general population were as follows. The null hypothesis that the average value of the series was 0 , in comparison with the alternative hypothesis, was rejected with a confidence level of $95 \%$. The sign test, based on counting the number of values above and below the assumed median, confirmed that the null hypothesis for the median of the series was rejected with a confidence level of $95 \%$. The sign rank test tested the null hypothesis that the median was 0 . It was based on comparing the average ranks of values above and below the assumed median. Since the probability value for this test was less than 0.05 , the null hypothesis could be rejected with a $95 \%$ confidence level. The sign and sign rank tests were less sensitive to the presence of outliers, but were somewhat less efficient than the t-test if all the data came from a single normal distribution.

Of particular interest was the analysis of standardized values of skewness and kurtosis that could be used to determine the origin of a sample from a normal distribution. The data output beyond the range from -2 to +2 indicated significant deviations from the norm, which could invalidate any statistical test related to standard deviation. In our case, the standardized values of skewness and kurtosis were within the range expected for data from the normal distribution.

Then, the confidence intervals for the mean and standard deviation of the passenger traffic series were investigated. The classical interpretation of these intervals says that in repeated sampling, these intervals will contain the true mean or standard deviation of the population (used for obtaining the data), in $95 \%$ of the cases. In our case, we were $95 \%$ confident that the interval for the true mean of the studied series was between 24104 and 38570 , and for the true standard deviation - between 7523 and 18 894. Both intervals allowed us to assume that the sampling population could be represented by a normal distribution. The confidence interval for the mean was fairly reliable and not very sensitive to challenges of this assumption, whereas the confidence interval for the standard deviation was quite sensitive. If the data were not obtained from a normal distribution, the standard deviation interval might be incorrect.

We also studied the estimated autocorrelations between the values of sets of passenger traffic at different lags. The lag autocorrelation coefficient $k$ measures the correlation between the values of a series at $t$ time and $(t-k)$ time. There was a $95 \%$ probability. In the case where the probability limits for a certain lag did not contain the estimated coefficient, there was a statistically significant correlation at this lag with a $95 \%$ confidence level. In our case, one of 24 autocorrelation coefficients was statistically significant at a $95 \%$ confidence level. This means that the time series could not be completely random ("white noise'). Measuring the value of this correlation coefficient, it can be argued that it showed an average rather than close link, that is why, with a certain degree of conditionality, it could be assumed that the results demonstrated the absence of autocorrelation in the studied data series. 
Forecasting was performed with the use of Statgraphics software. For obtaining time series data, five most appropriate forecasting models were selected, and analyzed qualitatively. Based on the visual analysis, the authors believe that the best models suited for time series forecasting include: linear and quadratic trend models; saturation curve models, linear exponential smoothing models (Holt's model), and ARIMA models. All the selected models showed an increase in passenger traffic. Their qualitative analysis on the basis of special tests was carried out in order to select the best model.

\section{Results and Discussion}

The use of statistical data on passenger traffic enables to forecast with adaptive and trend methods [10]. The application of these methods requires a certain number of observations for plotting a curve. It is assumed that the predictor variables that determined the past trend of the time series will similarly affect the final forecast. As a rule, the future period cannot be longer than the past period with available relevant numerical information. It is customary to obtain a forecast for a time period not exceeding one fourth of the base period. In addition, for the methods under consideration, the accuracy of the forecast decreases in the long run, hence, in our case, the forecast was generated for two subsequent periods.

The test data was used when choosing models and evaluating each model accuracy. Since the test data was not used in determining the forecasts, it could be a reliable indicator of how well the model is likely to forecast on new data. The quality analysis of the selected models was performed with special tests. Suitability of the model for predicting passenger numbers was performed by testing the behavior of residuals. First, the trough to peak ratio and trend reversals were detected. Then, the model was tested for the data sensitivity to the presence of a trend. For this purpose, the number of observations for which the estimated value of the predicted indicator was below or above the median was determined, i.e., it was investigated whether the number of deviations from the median was excessive. Since the data collected over a sufficient period of time are usually trended and affected by seasonal changes and other dimensions, observations for different time periods are autocorrelated. Given this feature, the Box Pierce test was used to test the assumption of overautocorrelation. The mean trend was tested to determine if there was a significant difference between the means. The variability trend was tested for significant variance difference. The results of the model testing are shown in Table 1.

Table 1. Results of testing forecasting models

\begin{tabular}{|l|c|c|c|c|c|}
\hline Model & $\begin{array}{c}\text { Test for } \\
\text { excessive } \\
\text { number of } \\
\text { peaks and } \\
\text { troughs }\end{array}$ & $\begin{array}{c}\text { Test for } \\
\text { excessive } \\
\text { deviations } \\
\text { from the } \\
\text { median }\end{array}$ & $\begin{array}{c}\text { Over- } \\
\text { autocorrelat } \\
\text { ion test }\end{array}$ & $\begin{array}{c}\text { Test for the } \\
\text { significance } \\
\text { of the mean } \\
\text { difference }\end{array}$ & $\begin{array}{c}\text { Test for the } \\
\text { significance } \\
\text { of the } \\
\text { variance } \\
\text { difference }\end{array}$ \\
\hline Linear Trend Model & $* *$ & OK & $*$ & OK & $*$ \\
\hline Quadratic Trend Model & $* *$ & OK & $* *$ & OK & OK \\
\hline Saturation Curve Model & OK & OK & OK & OK & OK \\
\hline $\begin{array}{l}\text { Linear Exponential } \\
\text { Smoothing Model } \\
\text { (Holt's Model) }\end{array}$ & OK & OK & OK & OK & $*$ \\
\hline ARIMA Model (1,0,0) & OK & OK & OK & OK & OK \\
\hline
\end{tabular}

Notes: OK - the model has passed the test

* The test fails at a confidence level of $95 \%$

$* *$ The test fails at a confidence level of $99 \%$

*** The test fails at a confidence level of $99.9 \%$. 
It is apparent from the data in Table 1 that at a confidence level of $95 \%$ or higher, only two models - the Saturation Curve and ARIMA models - are acceptable for the analyzed data.

Further, to assess the adequacy of the obtained dependencies, forecasting errors were evaluated using a variety of methods, namely, the root mean squared error; the mean error; the mean absolute error; the mean absolute percent error; and the mean percent error. The mean error is the difference between the measured and the aligned values: the closer the mean error goes to zero, the more accurate is the approximation. The root mean squared error and the mean absolute error are used to measure the difference between forecast and corresponding observed values. The mean percent error and the mean absolute percent error measure the accuracy of a forecast system as a percentage and can be calculated as the average absolute percent error for each time period minus actual values divided by actual values of passenger traffic. The values of the forecasting errors for five models are presented in Table 2 .

Table 2. Forecasting errors for five models

\begin{tabular}{|l|c|c|c|c|c|}
\hline \multicolumn{1}{|c|}{ Model } & $\begin{array}{c}\text { Root mean } \\
\text { squared error }\end{array}$ & $\begin{array}{c}\text { Mean } \\
\text { absolute } \\
\text { error }\end{array}$ & $\begin{array}{c}\text { Mean absolute } \\
\text { percent error }\end{array}$ & Mean error & $\begin{array}{c}\text { Mean percent } \\
\text { error }\end{array}$ \\
\hline Linear Trend Model & 2057.2 & 1544.0 & 4.8 & 0.0 & -0.2 \\
\hline $\begin{array}{l}\text { Quadratic Trend } \\
\text { Model }\end{array}$ & 1988.0 & 1528.4 & 5.4 & 0.0 & -0.5 \\
\hline $\begin{array}{l}\text { Saturation Curve } \\
\text { Model }\end{array}$ & 6307.9 & 4598.8 & 14.34 & 585.8 & -1.3 \\
\hline $\begin{array}{l}\text { Linear Exponential } \\
\text { Smoothing Model } \\
\text { (Holt's Model) }\end{array}$ & 2058.9 & 1506.5 & 4.44 & 306.78 & 0.3 \\
\hline $\begin{array}{l}\text { ARIMA Model } \\
\text { (1,0,0) }\end{array}$ & 2796.4 & 2183.9 & 8.0 & 1162.3 & 2.3 \\
\hline
\end{tabular}

As can be seen from Table 2, the ARIMA Model has lower error values as compared with the Saturation Curve Model. The visual analysis of the consistency of the obtained and forecast data also supports the benefits of the ARIMA Model for predicting a number of passengers.

The ARIMA Model combines an autoregressive model and a moving average model. ARIMA models are based on the iterative approach. The first step is to choose a model and apply a comparative analysis to time series forecasting. The focus is on residuals, in which case some constraints on the values of the parameters are required: the residuals do not contain useful information and are randomly distributed. The model parameters are estimated by minimizing the sum of squared errors by applying the nonlinear least squares method. If necessary, the procedure may be repeated to improve the quality of the model.

Figure 1 illustrates the correlation of the actual and forecast series data and the level change perspective. 


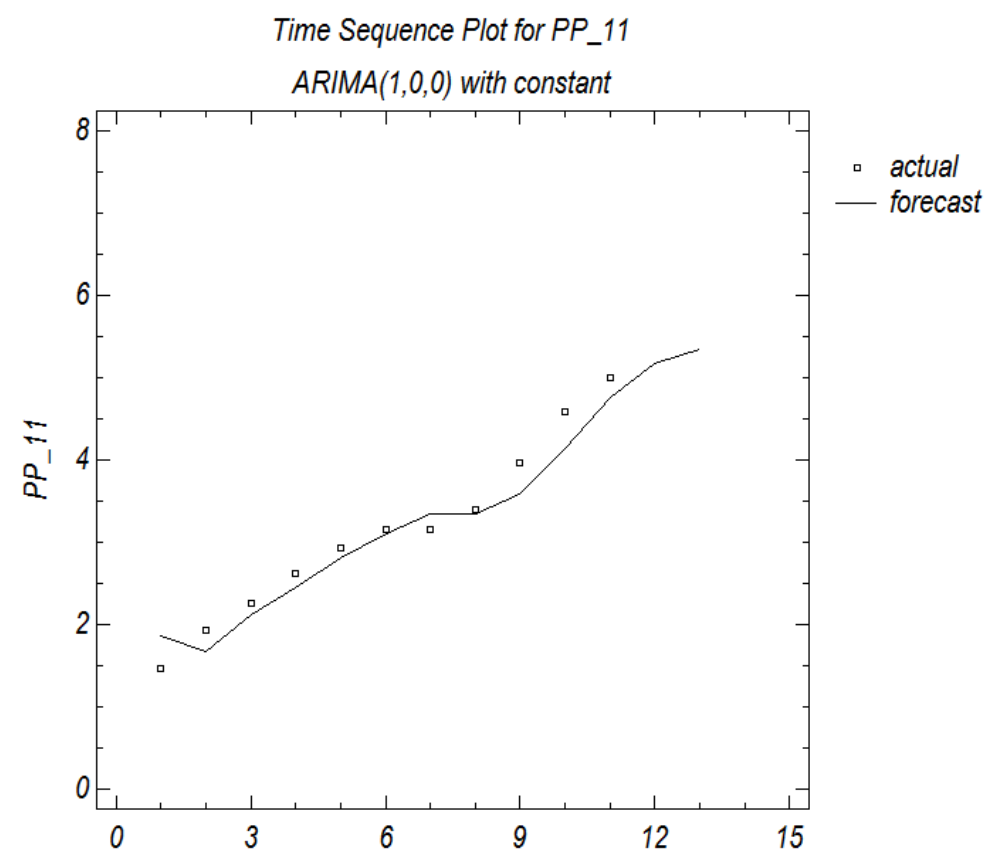

Fig. 1. Forecasts generated from the ARIMA model.

After eight iterations we were able to calculate the white noise variance with nine degrees of freedom equal to $9.4 \mathrm{E} 6$ and the standard deviation of white noise $(3070)$. The model predicts that the best forecast for future data will be provided by a parametric model that relates the most recent series data to the previous series data and noise. Statistical significance analysis demonstrated that all model members were statistically significant, since the probability of accepting the null hypothesis did not exceed $5 \%$.

According to the forecast, in 2020 the number of passengers was expected to increase to 51708 thou people, and in 2021 - to 53476 thou people. If the situation followed the most optimistic option, the forecast numbers could be 58655 thou and 63280 thou people, respectively.

In order to additionally assess the quality of the forecasting model, the training sample method was used. The method is to prepare a forecast on the basis of the reduced length of the data series and then compare the obtained forecast data with the true data. In our case, we used the data on passenger numbers for the period till 2017. It is worth noting that for the reduced series, all forecasting models passed the tests described above. However, the analysis of the forecast errors allows us to conclude that the ARIMA model is the most acceptable. The results of forecasting for the reduced series were compared with the actual data. The conclusion can be drawn that the true data are more consistent with the optimistic forecast of the training sample, i.e., it is possible to expect an increase in passenger traffic.

\section{Conclusion}

As it has been noted earlier, the forecasts were based on the assumption that the key predictor variables will continue affecting passenger numbers. However, due to the negative impact of COVID-19, regular international flights from Sheremetyevo International Airport were suspended in March 23, 2020, and steady growth in passenger 
traffic was replaced by a sharp drop. In 2021, the number of passenger has fallen by 2.6 times in comparison with 2019 and reached the figures of 2010.

As expected, the new time series data have significantly changed the outcomes of the forecast. A sharp change in the current trend has resulted in worse quality characteristics of the models. However, if we turn to the outcomes of the forecast, we can see that all forecasting models predict a future increase in passenger traffic in 2022 and 2023 to 22523 thou and 24446 thou people, respectively.

A similar pattern has been applied to forecast revenue of Sheremetyevo International Airport. The quality of the series has been tested. Checking the statistical data on the amount of revenue enables to use adaptive and trend forecasting methods. As for the passenger traffic series, the five most acceptable forecasting models were selected: the Linear Trend Model, the Quadratic Trend Model, the Exponential Trend Model, the Linear Exponential Smoothing Model (Holt's Model), and the ARIMA model. All selected models showed revenue growth. To choose the final model, a qualitative analysis was performed based on the above mentioned tests and the values of the forecast errors. The results of the tests give grounds to assert that Holt's Model is best suited for the analyzed data at the level of reliability of $95 \%$.

If there is an upward trend in the time series, then along with the assessment of the current level of the series, an assessment of the slope is also necessary. Holt's method allows an estimate of the level of the series and an estimate of the trend (slope) smooth directly, with different smoothing constants for each of them. These smoothing constants enable to measure the current level and slope and adjust them with every new observation. An important benefit of Holt's method is its flexibility, which gives an opportunity to choose the ratio which tracks the level of the series and its slope (trend). Though the choice of smoothing parameters may be subjective, the authors aimed to minimize forecasting errors.

According to the results of the forecast, in 2020 the revenue was expected to rise to 86 291 million rubles, and in 2021 - to 95793 million rubles. The most likely forecast scenario is that the revenue might amount to 102538 and 117109 million rubles, respectively.

In order to additionally assess the quality of the forecasting model, the training sample method was used. The revenue till 2017 was taken as the forecasting base. The resulting forecasts generated from Holt's model fully agree with the true data. Figure 2 shows the correlation of actual and forecast series data generated from Holt's method and possible changes in the series levels. 
Time Sequence Plot for $V_{-} 11$

Holt's linear exp. smoothing with alpha $=0,7254$ and beta $=0,171$

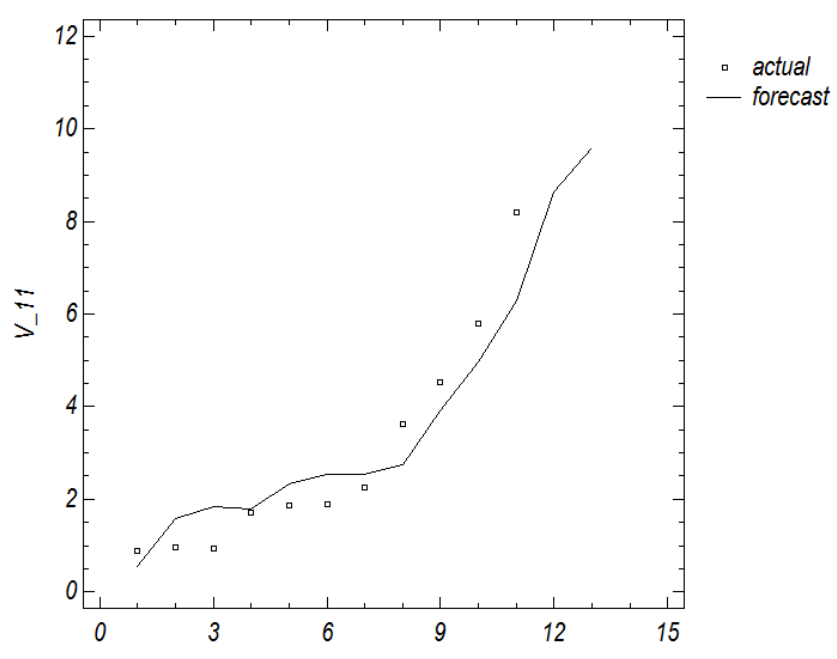

Fig. 1. Forecasts generated from Holt's method

Similar to the passenger traffic statistics, the steady and sustained revenue growth was replaced by a sharp drop. In 2021, the revenue decreased four-fold compared to 2019 and reached the level comparable with the 2014 revenue. The decline in revenue as a result of the imposed restrictions significantly changed the resulting forecasts and lowered the predictive quality of the models, although even in this case, revenue growth is predicted as the most likely sequence of events. In the next two years, the revenue might rise to 56334 million rubles.

To sum up: air transport service is unlike to leave the market, but will adjust to the realities of the post-COVID market. At the moment, it is difficult to predict how long the isolation will continue. The longer it lasts, the more serious its consequences might be. The proposed forecasting models can be successfully used when new data on revenue and passenger traffic are received, or information on the impact of coronavirus is updated nationally and internationally. With re-established transport networks between countries, forecasting with the application of adaptive methods may quite accurately predict trends in passenger traffic [11].

The aim of the study was to provide comprehensive assessment of the current state and future prospects for the development of air transport that plays a significant role in the country's economy. The main limitation of the studied forecasting methods is that they consider the time series in isolation from other phenomena, and even if additional information is available, it can only be used by adjusting the adaptation rate. At the same time, provided that the studied dynamics of the indicator is not subject to sharp changes in trends caused by the impact of random and unpredictable factors (e.g. the COVID pandemic), these models offer sensible results that can be used for making managerial decisions at various levels.

\section{References}

1. D. Ivanov, International journal of logistics management, 1 (2021)

2. E.L. Molokova, Modern Science, 12-3 (2019) 
3. M.G. Chung, A. Herzberger, J. Liu, K.A. Frank, Journal of travel research, 59-3 (2020)

4. M.P. Bonser, International Journal of Aviation, Aeronautics, and Aerospace, 6-3 (2019)

5. E.G. Animitsa, I.I. Rakhmeeva, Social Sciences and Modernity, 6 (2020)

6. A.A. Laughs, Marketing models of the transport market (1998)

7. S.V. Orekhova, E.V. Kislitsyn, Espacios, 40/6 (2019)

8. Z.E. Konakbay, I.Zh. Asilbekova, Bulletin of the Academy of Civil Aviation, 4(19) (2020)

9. E.M. Kochkina, E.V. Radkovskaya, M.V. Drobotun, Bulletin of the Ural State University of Economics, 2(64) (2016)

10. I.V. Naumov, Yu. V. Dubrovskaya, Ye. V. Kozonogova, Economy of the region, 16(3) (2020)

11. V.G. Afanasyev, Scientific Bulletin of the Moscow State Technical University of Civil Aviation, 214(4) (2015) 